05

\title{
Модельный расчет высокоскоростного соударения тел различной формы с массивными металлическими преградами
}

\author{
(ㄱ А.С. Власов, А.Б. Синани \\ Физико-технический институт им. А.Ф. Иофрфе РАН, \\ 194021 Санкт-Петербург, Россия \\ e-mail: alexandervlasov@yandex.ru
}

(Поступило в Редакцию 11 июля 2016 г.)

\begin{abstract}
Представлена математическая модель высокоскоростного соударения тел произвольного удлинения с массивными металлическими преградами. В основу модели положены баланс энергии и поэтапная схема образования кратера. Процесс соударения рассматривается как квазистационарное проникание деформирующегося ударника и инерционное углубление кратера на заключительном этапе. Сопоставление расчетов с результатами численного моделирования и физического эксперимента продемонстрировало удовлетворительное совпадение.
\end{abstract}

DOI: 10.21883/JTF.2017.07.44674.1992

\section{Введение}

Задача конечной баллистики формулируется следующим образом. В момент $t=0$ происходит соударение двух тел (ударника и преграды). Необходимо определить дальнейшее движение среды. Под термином определить подразумевается либо измерить (эксперимент), либо рассчитать (теория).

В экспериментальных исследованиях измеряется скорость соударения, определяются размеры кратера в преграде и деформация ударника. В процессе эксперимента также могут фиксироваться временные зависимости проникания и деформации ударника, скорость свободной поверхности преграды, иногда положение фронта ударной волны. Фактически экспериментальная информация сводится к геометрическим и кинематическим характеристикам. Практически невозможно непосредственно получить какую-либо информацию о силовых характеристиках процесса (измерять напряжения в зоне взаимодействия соударяющихся тел).

Фактически максимум, что можно получить из экспериментальных данных без привлечения теоретических представлений - это некоторые аппроксимирующие зависимости между измеренными величинами (например, скорость удара-размеры кратера).

Строгие теоретические расчеты встречают значительные трудности ввиду сложности рассматриваемых задач. Вследствие этого широкое распространение получили численные методы решения задач конечной баллистики [1-5]. Следует отметить, что такой подход скорее следует отнести к специфическому виду экспериментов (вычислительному эксперименту), поскольку он не решает основную задачу теории - находить взаимосвязь между характеризующими изучаемое явление параметрами.

В связи с этим сохраняют актуальность задачи разработки упрощенных теоретических подходов к широкому кругу задач высокоскоростного взаимодействия тел. В настоящее время область высокоскоростного удара привлекает пристальное внимание исследователей. Этот интерес стимулируется развитием космических и оборонных технологий.

В настоящей работе представлен вариант упрощенной модели высокоскоростного удара по массивным однородным металлическим преградам.

\section{Основные положения модели}

Дадим качественное определение высокоскоростного удара и тем самым очертим область применимости модели. Соударение можно считать высокоскоростным, если его результат отвечает следующим условиям:

1. Объем кратера в преграде сушественно превышает объем ударника.

2. Ударник полностью деформируется и растекается в тонкую пелену по стенкам кратера.

В основу модели традиционно положено уравнение баланса энергии. Кинетическая энергия ударяющего тела расходуется на необратимое деформирование преграды (образование кратера) и самого ударника. Остальными затратами энергии можно пренебречь. Удельная энергия деформирования $\varepsilon_{p}$ (на единицу объема) ударника слабо зависит от скорости удара [6] и может быть принята постоянной величиной. В качестве оценки $\varepsilon_{p}$ принято $Y_{p} \varepsilon\left(Y_{p}\right.$ - динамический предел текучести при сжатии, эффективная деформация ударника $\varepsilon \sim 1)$. Образующийся в результате высокоскоростного удара кратер имеет цилиндрическую форму с полусферическим дном. В частных случаях кратер может вырождаться в полусферу и даже сферический сегмент.

Объем кратера пропорционален энергии, затраченной на его образование. При этом предполагаем, что коэффициент пропорциональности $\varepsilon_{t}$ (имеет смысл удельной энергии деформирования материала преграды) определяется только механическими свойствами преграды и не зависит от свойств ударника и скорости удара. 
Эти положения позволяют сформулировать первое уравнение модели, связывающее кинетическую энергию ударника и объем образующегося кратера,

$$
\frac{1}{2} \rho_{p} L_{0} r_{0}^{2}\left(V_{i}^{2}-\frac{2 \varepsilon_{p}}{\rho_{p}}\right)=\varepsilon_{t}\left(P_{c}-\frac{1}{3} R_{c}\right) R_{c}^{2}
$$

Поскольку при сделанных предположениях кратер характеризуется двумя величинами - глубиной $\left(P_{c}\right)$ и радиусом $\left(R_{c}\right)$, - необходимо еще одно соотношение.

В качестве второго соотношения используем уравнение для глубины кратера. Качественной схема процесса соударения была сформулирована еще в 60-х годах прошлого века [7]. В соответствии с этой схемой процесс проникания ударника в преграду в общем случае представляет последовательность трех этапов.

1. Начальный нестационарный этап формирования деформированной головной части.

2. Дальнейшее квазистационарное проникание и деформирование ударника.

3. Заключительный этап инерционного движения материала преграды после полной деформации ударника, приводящего к формированию окончательной конфигурации кратера в преграде.

Следует отметить, что на начальной фазе соударения происходят чрезвычайно резкие изменения как по величине, так и по характеру напряженного состояния в голове стержня (взаимодействия ударных волн и волн разгрузки в первые моменты после соударения). Однако эти изменения значительно слабее отражаются на кинематических характеристиках процесса. Поэтому можно принять, что средняя скорость внедрения на начальном нестационарном этапе незначительно отличается от последующего квазистационарного значения, и можно рассматривать в рамках единого квазистационарного процесса оба этапа.

Таким образом, глубина кратера определяется квазистационарным прониканием ударника и углублением кратера вследствие инерционного течения на заключительном этапе. Квазистационарное соударение удлиненных тел активно исследуется на протяжении более полувека. Первая теоретическая модель квазистационарного внедрения (модель Алексеевского-Тейта) предложена в [8-10]. Впоследствии предложены как уточнения данной модели, так и альтернативные подходы [11-17]. Следует отметить, что модель Алексеевского-Тейта $(\mathrm{A}-\mathrm{T})$ до настоящего времени остается по популярности вне конкуренции.

Кратко рассмотрим основные особенности данной модели. Процесс высокоскоростного соударения деформируемого тела с преградой описывается следующим минимальным набором характеристик: $U-$ скорость внедрения (скорость осевой точки контактной границы ударник/преграда), $V$ - скорость недеформированного остатка (заднего торца) ударника, $L-$ длина ударника, $P$ - глубина внедрения в преграду.
Система уравнения модели имеет следующий вид:

$$
\begin{gathered}
\frac{d P}{d t}=U, \\
\frac{d L}{d t}=-(V-U), \\
\frac{d V}{d t}=-\frac{Y_{p}}{\rho_{p}(L-h)},
\end{gathered}
$$

где $h$ - протяженность пластически деформированной зоны в голове стержня.

Для взаимосвязи скоростей $U$ и $V$ используем так называемое модифицированное уравнение Бернулли (выводится из уравнения сохранения момента в стационарном приближении)

$$
\frac{1}{2} \rho_{p}(V-U)^{2}+R_{p}=\frac{1}{2} \rho_{t} U^{2}+R_{t}
$$

В уравнении фигурируют два параметра $R_{p}$ и $R_{t}$ (прочностные сопротивления ударника и преграды соответственно), характеризующие прочностные свойства материалов взаимодействующих тел.

Следует отметить, что уравнение (4) остается физически корректным при длине стержня более 2-3 диаметров. Строго говоря, модель можно использовать для расчета глубины внедрения в преграду только в предельном случае бесконечно тонкого стержня $(d \rightarrow 0)$. Тогда глубина внедрения, пропорциональная длине стержня, конечна, а вклад нестационарных этапов, пропорциональный диаметру, пренебрежимо мал.

Инерционное движение дна кратера на заключительном этапе взаимодействия имеет качественную аналогию с расширением сферической полости в несжимаемой бесконечной среде.

Рассмотрим следующую задачу. Сферическая полость радиуса $R_{0}$ нагружается в момент времени $t=0$ импульсом давления. Вследствие несжимаемости среды ударная волна мгновенно уходит на бесконечность, оставляя за собой распределение скоростей. Рассматриваем последующее движение среды, полагая, что работа пластического деформирования приводит к уменьшению кинетической энергии материала среды, т.е.

$$
d W+d E=0
$$

где $d W$ - приращение работы пластического деформирования и $d E-$ изменение при этом кинетической энергии материала среды.

Кинетическая энергия материала, заключенного в сферическом слое толщиной $d r$, равна

$$
\frac{1}{2} \rho u^{2} 4 \pi r^{2} d r=2 \pi \rho_{t} U^{2} R^{4} \frac{d r}{r^{2}} .
$$

Здесь использовано условие несжимаемости $U R^{2}=$ $=u(r) r^{2}, U=U(R)$ - скорость стенок полости. 
Интегрируя это выражение от $R$ до бесконечности, получим кинетическую энергию в материале среды с полостью радиуса $R$ :

$$
E=2 \pi \rho R^{3} U^{2} .
$$

При смещении границы полости на $d R$ изменение кинетической энергии равно

$$
d E=2 \pi \rho\left(3 R^{2} U^{2} d R+2 R^{3} U d U\right) .
$$

Приращение работы пластического деформирования при этом равно

$$
d W=\varepsilon 4 \pi R^{2} d R,
$$

где $\varepsilon-$ энергия пластического деформирования среды при увеличении объема полости на единицу.

Подставляя полученные выражения в уравнение баланса энергии, получаем следующее обыкновенное дифференциальное выражение:

$$
\frac{2}{3} R U \frac{d U}{d R}+U^{2}+v_{0}^{2}=0,
$$

решение которого дает зависимость скорости движения стенки от текущего радиуса полости

$$
U=v_{0} \sqrt{\left(R_{c} / R\right)^{3}-1},
$$

где $v_{0}=\left(\frac{2 \varepsilon}{3 \rho}\right)^{1 / 2}$, а $R_{c}-$ максимальный радиус полости, получаемый из условия $U=0$.

Предполагается, что к моменту полной деформации ударника дно кратера вблизи оси симметрии можно аппроксимировать частью сферической поверхности радиуса $R_{0}$. Дальнейшее перемещение дна кратера рассматривается как инерционное движение сферической полости от начального радиуса $R_{0}$ до конечного $R_{c}$. Начальный радиус полости определяется из условия равенства скорости стенки полости скорости внедрения $U$ в этот момент.

Рассмотрим вопрос „склейки“ двух решений — квазистационарного проникания и инерционного расширения. Очевидно, что окончание одного этапа и начало следующего не совпадают ни в пространстве, ни во времени. Поэтому необходимо выбрать некоторую фиктивную границу, разделяющую два этапа и продлить решения до этой границы.

Для этого преобразуем уравнения модели А-Т. В качестве независимой переменной примем укорочение ударника $x=L_{0}-L$. Тогда система уравнений для $P, V$, $U$ примет следующий вид:

$$
\begin{gathered}
\frac{d P}{d x}=\frac{U}{V-U}, \\
\frac{d V}{d x}=\frac{Y_{p}}{\rho_{p} L_{0}\left(1-x / L_{0}\right)} \frac{1}{(V-U)} .
\end{gathered}
$$

В последнем уравнении мы пренебрегли высотой головной части $h$. „Модифицированное уравнение Бернулли“ остается неизменным. Разложим $1 /\left(1-x / L_{0}\right)$ в ряд, сохранив члены до третьего порядка включительно. Тогда уравнение (14) примет следующий вид:

$$
\frac{d V}{d x}=\frac{Y_{0}}{\rho_{p} L_{0}}\left(1+x / L_{0}+\left(x / L_{0}\right)^{2}+\left(x / L_{0}\right)^{3}\right)\left(\frac{1}{V-U}\right) .
$$

Преобразованное уравнение (15) позволяет получить формальное решение вплоть до нулевой длины ударника. Это решение практически совпадает с решением исходной системы, за исключением предельно больших укорочений стержня. Будем считать, что продолжение решения до „полной“ деформации стержня в какой-то степени учитывает переход между этапами.

Если пренебречь торможением ударника, то получаем очевидное решение: глубина кратера при полной деформации ударника равна

$$
P_{1}=P\left(x=L_{0}\right)=\frac{U}{V-U} L_{0} .
$$

Дальнейшее движение дна кратера рассматриваем как расширение сферической полости от начального радиуса $R_{0}=\frac{R_{c}}{\left(1+U^{2} / v_{0}^{2}\right)^{1 / 3}}$ до конечного радиуса кратера.

Приращение глубины кратера на заключительном этапе равно

$$
R_{c}-R_{0}=R_{c}-\frac{R_{c}}{\left(1+U^{2} / v_{0}^{2}\right)^{1 / 3}} .
$$

В итоге получаем для глубины кратера следующее выражение:

$$
P_{c}=P_{1}+\left[1-\frac{1}{\left(1+U^{2} / v_{0}^{2}\right)^{1 / 3}}\right] R_{c} .
$$

При учете торможения ударника для получения $P_{1}$ необходимо решить систему уравнений (13), (15) и (5). Следует отметить, что торможение дает достаточно малый вклад в результаты расчетов даже на нижней границе области высокоскоростного удара.

Таким образом, мы пришли к системе двух алгебраических уравнений (1) и (18) для определения конечных размеров кратера, образованного в преграде в результате высокоскоростного удара. Исходными данными являются размеры ударника, плотности материалов ударника и преграды и скорость соударения. Используются следующие прочностные характеристики - удельная энергия деформирования $\varepsilon_{t}$ и прочностные члены в ,модифицированном уравнении Бернулли“ $R_{t}, R_{p}$. „Прочностные сопротивления“ появляются только в виде разности $R_{t}-R_{p}$.

Сделаем несколько замечаний по представленной модели.

1. В случае удара стержня большого удлинения процесс радиального расширения цилиндрической части кратера может закончиться прежде, чем сформируется его дно. Рассчитывать радиус кратера, исходя из его 
объема, в такой ситуации представляется логически противоречивым. Однако рассчитанный размер практически будет совпадать с радиусом бесконечно глубокого кратера, образованного в результате удара бесконечно длинного ударника. Последний же случай не вносит никаких логических противоречий. Можно предполагать, что наиболее эффективно предложенная расчетная схема должна работать в следующей ситуации - быстрая (почти мгновенная) передача энергии ударника преграде + существенно более длительное течение последней. То есть длительность полного деформирования ударника должна в идеале составлять малую часть времени соударения.

2. Для компактных ударников $\left(L_{0} / d_{0} \sim 1\right)$ не имеет смысла говорить об квазистационарном процессе. Поэтому нет обоснованных теоретических оснований для использования модифицированного уравнения Бернулли при расчете скорости внедрения. В настоящий момент просто принимается, что нестационарными эффектами можно пренебречь. Это позволяет дать разумную оценку величины скорости внедрения компактного ударника.

3. При расчетах ударник всегда является прямым круговым цилиндром с плоскими торцами. Все другие конфигурации пересчитываются на „эквивалентный цилиндр“, имеющий ту же массу и удлинение, что и оригинальный ударник. Например, для широко используемых в баллистических экспериментах ударников сферической формы размеры ,эквивалентного цилиндра“ $L_{e q}=d_{e q}=(2 / 3)^{1 / 3} d_{s p h}$.

4. При выводе уравнения для глубины кратера фактически построена зависимость скорости внедрения $u$ от текущего положения ударника в преграде $z$. Следовательно, построение временных характеристик процесса внедрения является чисто технической проблемой - peшением обыкновенного дифференциального уравнения $d z / d t=u(z)$.

\section{Соударение тел из одинаковых материалов}

В качестве первого примера были проведены расчеты соударения цилиндрических ударников различного удлинения с массивными преградами. Материал Al 1100-0 (технически чистый алюминий). Для сравнения использованы результаты численного моделирования [18], а также результаты экспериментов [19].

Была рассчитана удельная энергия деформирования $\varepsilon_{t}$ как отношение кинетической энергии ударника к объему кратера. Для всех типов ударников $\varepsilon_{t}$ не зависит от скорости соударения. Для удлиненных (начиная с $L / d=2$ ) ударников удельная энергия деформирования постоянна и равна $\varepsilon_{t}=0.60 \mathrm{~kJ} / \mathrm{cm}^{3}$. Постоянство $\varepsilon_{t}$ достаточно очевидно в предельном случае для глубоких туннелеобразных кратеров. Интересно отметить, что практически такое же значение получено при скорости удара выше $11 \mathrm{~km} / \mathrm{s}$ (разгон стержня кумулятивным зарядом) [19] и в случае внедрения недеформированного ударника со скоростью несколько сот $\mathrm{m} / \mathrm{s}$.

В случае компактных ударников наблюдается существенное увеличение данной величины. Можно предположить, что увеличение $\varepsilon_{t}$ имеет фиктивный характер. Объем кратера стандартно отсчитывается от уровня исходной неискаженной поверхности преграды. Искажение же поверхности преграды может быть значительным. Таким образом, при измерении объема отбрасывается некоторая часть, фактически относящаяся к кратеру. В итоге получаем завышенное значение $\varepsilon_{t}$. Это завышение пренебрежимо мало для глубоких кратеров, но может оказаться существенным для мелких. По-видимому, применение предлагаемой расчетной схемы целесообразно ограничить ударниками с удлинением не менее $L / d \sim 1$.

В расчетах учитывался эффект зависимости $\varepsilon_{t}$ от формы кратера $f_{c}\left(P_{c} / D_{c}\right)$.

На рис. 1 приведено сравнение расчетов с результатами численного моделирования [18] и данными экспериментов [20]. В расчетах использовано значение прочностного сопротивления $R_{t}-R_{p}=0.60 \mathrm{GPa}$.

На рис. 2 приведены результаты расчетов и экспериментов по соударению сферических ударников с алюминиевой преградой $(99.99 \%$ алюминий $)$ [21,22]. Данные нормированы на диаметр шара. Невзирая на достаточно большой разброс данных, совпадение можно считать удовлетворительным. Эти расчеты подтверждают возможность использования предложенной модели для оценки внедрения компактных ударников.

В качестве третьего примера рассчитаны результаты соударения стального цилиндрического ударника $(L / d=3)$ со стальной массивной преградой из мягкой стали. Твердость стали по Бринелю составляла $H_{B}=1.1-1.3 \mathrm{GPa}$. Значение удельной энергии деформирования определено из экспериментальных данных по объему кратера в преграде [20].

Зависимость глубины кратера от скорости соударения представлена на рис. 3. Совпадение с экспериментом [20] можно признать хорошим. Интересно сопоставить эти результаты с результатом для алюминия, приведенным выше на рис. 1. Естественно, зависимости для стали и алюминия не совпадают. Однако нетрудно показать, что должно иметь место следующее соотношение: $P_{c} / d=F\left(L / d, V_{i} /\left(\varepsilon_{t} / \rho_{t}\right)^{1 / 2}\right)$. Данная безразмерная зависимость для алюминия и стали показана на рис. 4 . Наблюдается практическое совпадение обеих кривых.

\section{Соударение тел из неодинаковых материалов}

Обратимся к более сложному высокоскоростному случаю соударения тел из неодинаковых материалов. Представленная модель предсказывает, что с увеличением скорости удара форма кратера достаточно быстро приближается к полусферической (т. е. отношение глубины 

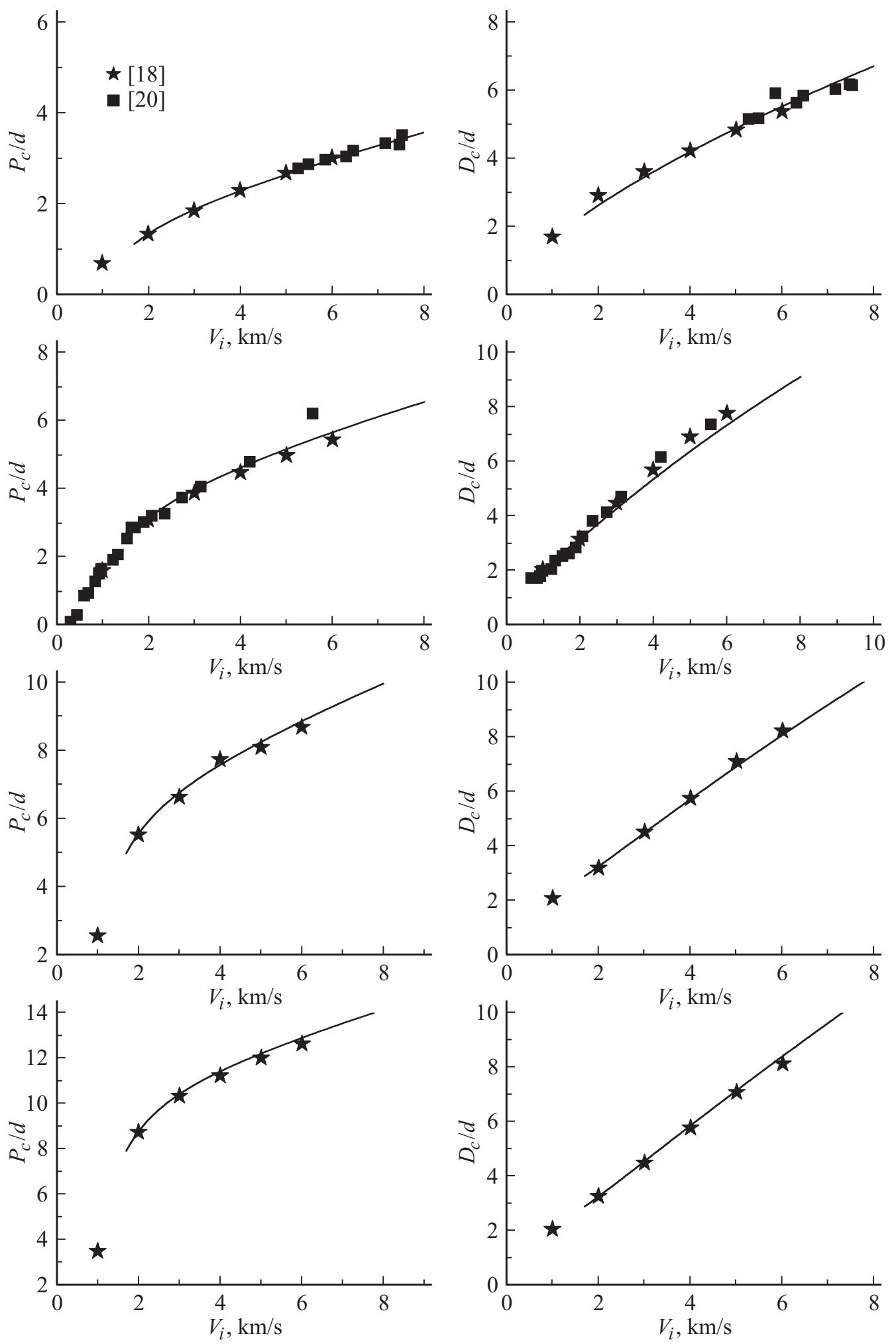

Рис. 1. Зависимость размеров кратера от скорости соударения (сверху вниз $-L / d=1,3,6,10)$.

кратера к его диаметру должно быть $~ 0.5)$. Между тем эксперименты показывают, что при ударе более плотным компактным телом по преграде меньшей плотности образуется кратер, несколько вытянутый в направлении удара по сравнению с полусферическим. Численное мо- делирование подтверждает, что этот эффект сохраняется вплоть до экстремально высоких скоростей $[23,24]$.

Для того чтобы учесть эти особенности, необходимо внести определенные изменения в предлагаемую модель. Используя аргументы теории размерностей, мож- 
но предположить, что форма кратера определяется в первую очередь следующими безразмерными параметрами: $\rho_{p} / \rho_{t}, \rho_{t} V^{2} / \varepsilon_{t}, R_{t} / R_{p}$. Поскольку форма кратера слабо зависит от скорости удара, можно допустить, что она в первую очередь определяется отношением плотностей материалов ударника и преграды. В качестве простейшей функции можно выбрать $\left(\rho_{p} / \rho_{t}\right)^{q}$, где $q-$ постоянная. Представим дно кратера в виде полуэллипсоида (точнее, псевдополуэллипсоида), отношение
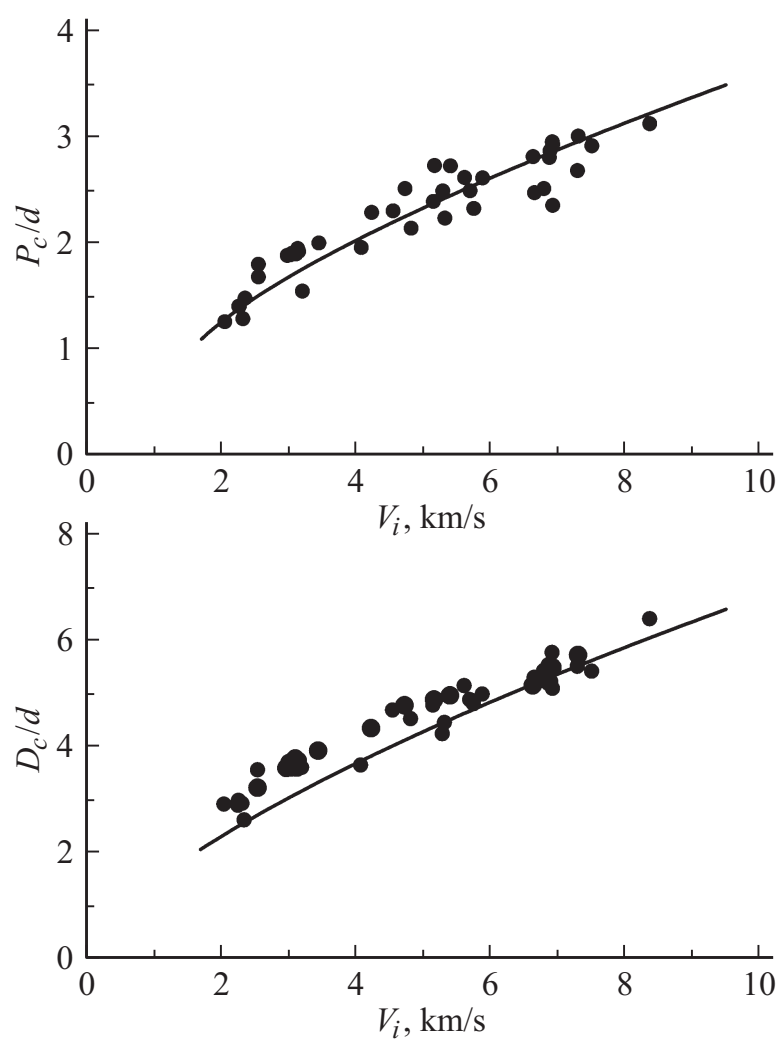

Рис. 2. Зависимость $P_{c} / d$ и $D_{c} / d$ от $V_{i}$.

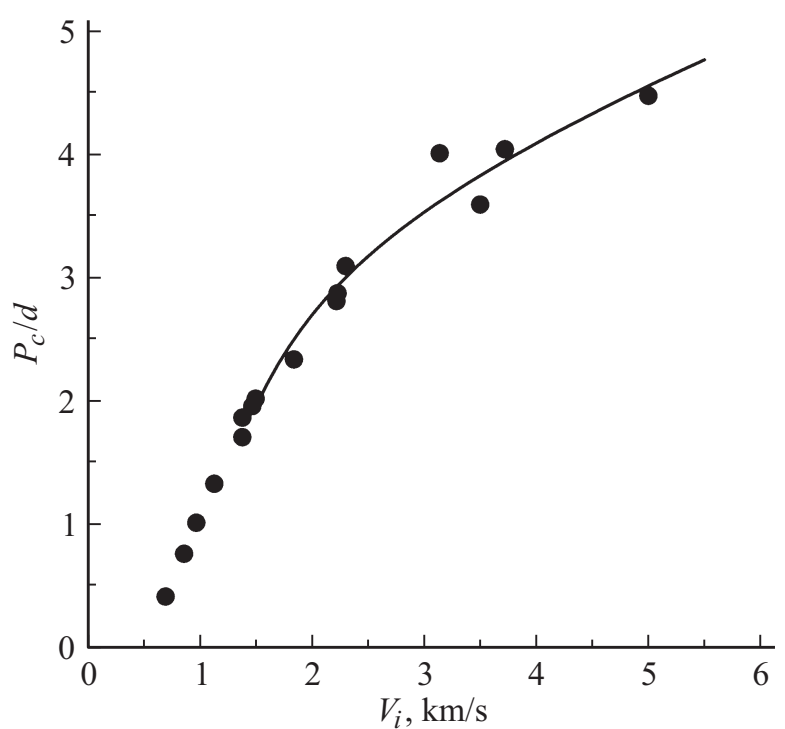

Рис. 3. Зависимость $P_{c} / d$ от $V_{i}$.

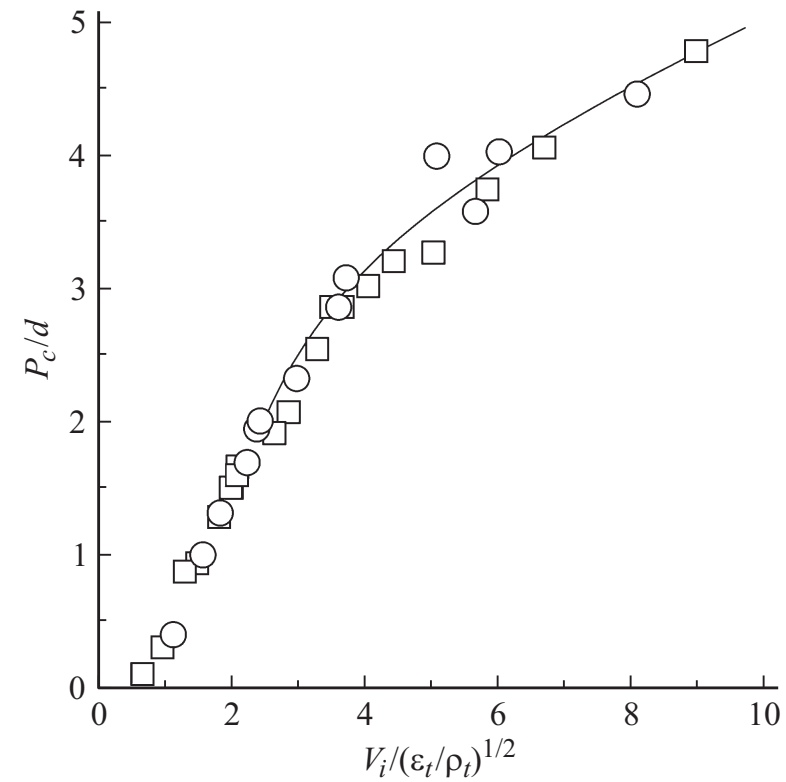

Рис. 4. Зависимость $P_{c} / d$ от $V_{i} /\left(\varepsilon_{t} / \rho_{t}\right)^{1 / 2}$.

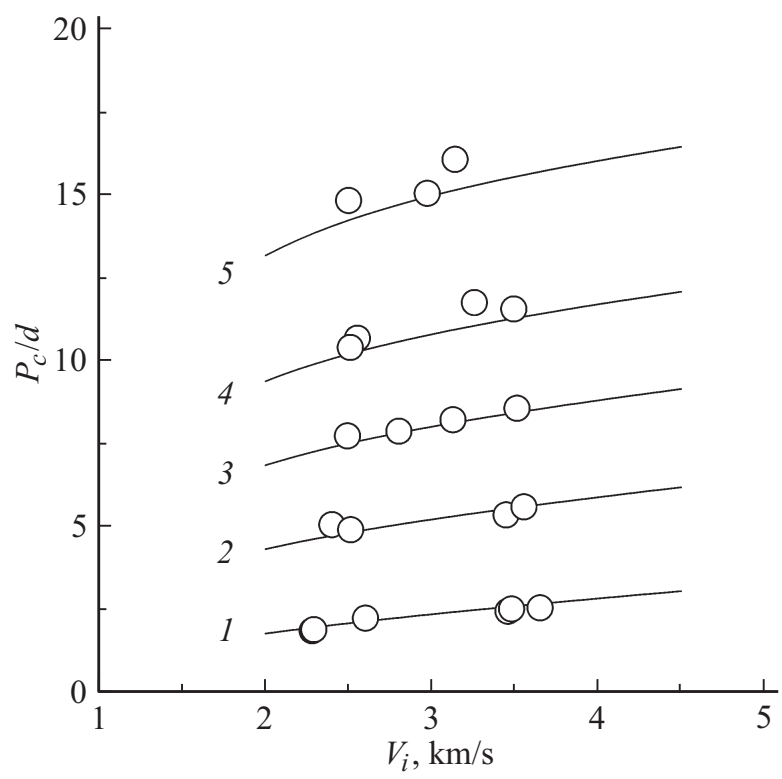

Рис. 5. Зависимость $P_{c} / d$ от $V_{i}: 1-L / d=1,2-3,3-5$, $4-7,5-10$.

полуосей которого зависит от $\rho_{p} / \rho_{t}$. Поверхность дна такого кратера в цилиндрических координатах описывается следующим уравнением: $r=b \sqrt{1-\left(z / R_{c}\right)^{Q}}$, а объем $w=\frac{Q}{Q+1} \pi a b^{2}$. Сопоставление с численным расчетом [25] позволило представить $Q$ как функцию отношения плотностей: $Q=2+\left[\left(\rho_{p} / \rho_{t}\right)^{0.37}-1\right]$.

Уравнения (1) и (18) приобретают следующий вид:

$$
\begin{gathered}
\frac{1}{2} \rho_{p} L_{0} r_{0}^{2}\left(V_{i}^{2}-\frac{2 \varepsilon_{p}}{\rho_{p}}\right)=\varepsilon_{t}\left(P_{c}-\frac{1}{Q+1} R_{c}\right) R_{c}^{2}, \\
P_{c}=P_{1}+\left[1-\frac{1}{\left(1+U^{2} / v_{0}^{2}\right)^{1 / 3}}\right] q R_{c} .
\end{gathered}
$$


Цилиндрический кратер с полусферическим дном является частным случаем $\left(\rho_{p}=\rho_{t}\right), Q=2$. По-видимому, наиболее важной парой неодинаковых металлических материалов является вольфрамовый сплав и броневая сталь. Высокоскоростному соударению данной пары посвящено множество исследований.

На рис. 5 приведены результаты расчетов глубины внедрения $\left(P_{c} / d\right)$ цилиндрических ударников из вольфрамового сплава в броневую сталь твердостью $H_{B}=2.95 \mathrm{GPa}[26]$.

Использованные при расчете характеристики материалов: $\rho_{p}=17.6 \mathrm{~g} / \mathrm{cm}^{3}, \rho_{t}=7.85 \mathrm{~g} / \mathrm{cm}^{3}, \varepsilon_{t}=4.5 \mathrm{~kJ} / \mathrm{cm}^{3}$, $R_{t}-R_{p}=2.0 \mathrm{GPa}, \varepsilon_{t}-$ экспериментальное значение. Прочностное сопротивление было рассчитано по результатам численного моделирования [27] процесса внедрения стержня из вольфрамового сплава в преграду из стали 4340. Эта сталь по механическим свойствам очень близка к броневой стали. Наблюдается вполне удовлетворительное совпадение результатов для всех удлинений ударников.

\section{Заключение}

Представлена приближенная теоретическая модель для расчета параметров высокоскоростного соударения ударников произвольного удлинения с массивными металлическими преградами. Сопоставление приближенных расчетов с результатами численного моделирования и физического эксперимента продемонстрировало удовлетворительное совпадение. В большинстве случаев расхождение оказывается меньше 10\%.

\section{Список литературы}

[1] Wilkins M.L. Computer simulation of Dynamic Phenomena. Springer, 1999. $265 \mathrm{p}$.

[2] Zukas J.A., Segletes S.B. // Adv. Eng. Software. 1992. Vol. 14. P. 77-84.

[3] Sedgwick R.T., Hageman L.J., Herrmann R.G., Waddell J.L. // Int. J. Eng. Sci. 1978. Vol. 16. N 11. P. 859-869.

[4] Johnson W.E., Anderson C.E., Jr. // Int. J. Impact. Engng. 1987. Vol. 5. N 1-4. P. 429-439.

[5] Бабкин А.В., Колпаков В.И., Охитин В.Н., Селиванов В.В. Численные методы в задачах физики быстропротекающих процессов. М.: Изд-во МГТУ им. Н.Э. Баумана, 2006. $520 \mathrm{c}$.

[6] Anderson C.E., Jr., Littlefield D.L., Walker J.D. // Int. J. Impact Engng. 1991. Vol. 11. N 1. P. 1-12.

[7] Christman D.R., Gehring J.W. // J. Appl. Phys. 1966. Vol. 37. N 4. P. $1579-1587$.

[8] Алексеевский В.П. // Физика горения и взрыва. 1966. Т. 2. Вып. 2. С. 99-106.

[9] Tate A. // J. Mech. Phys. Sol. 1967. Vol. 15. N 6. P. 387-399.

[10] Tate A. // J. Mech. Phys. Sol. 1969. Vol. 17. N 3. P. 141-150.

[11] Perez E. // Science et Techniques de l'Armement. 1982. Vol. 56. P. 1-155.

[12] Jones S.E., Gillis P.P., Foster J.C., Jr. // J. Mech. Phys. Sol. 1987. Vol. 35. N 1. P. $121-131$.
[13] Jones S.E., House J.W., Wilson L.L., Cinnamon J.D. // Int. J. Impact Engng. 1992. Vol. 12. N 2. P. 145-166.

[14] Yaziv D., Riegel J.P. // Int. J. Impact Engng. 1993. Vol. 14. N 1-4. P. 843-850.

[15] Luk V.K., Piekutowsli A.J. // Int. J. Impact Eng. 1993. Vol. 11. N 3. P. 323-340.

[16] Roisman I.V., Yarin A.L., Rubin M.B. // Int. J. Impact Engng. 2002. Vol. 25. N 6. P. 573-597.

[17] Rubin M.B., Yarin A.L. // Int. J. Impact Engng. 2002. Vol. 27. N 4. P. 387-398.

[18] Mc Henry M.R., Choo Y., Orphal D.L. // Int. I. Impact Engng. 1999. Vol. 23. N 1. Part 2. P. 621-628.

[19] Walker J.D., Grosch D.J., Mullin S.J. // Int. J. Impact Engng. 1995. Vol. 17. N 4-6. P. 903-914.

[20] Anderson C.E., Morris B.L., Littlefield D.L. A Penetration Mechanics database. // SwRI Report 9593/C01. 1990.

[21] Olive G., Engel O.G. // Proc. of the $6^{\text {th }}$ Symposium on Hypervelocity Impact. 1963. Vol. II. Part 2. P. 337-366.

[22] Liles C.D., Goodman E.H. // Tech. Rep. N AEDC-TOR-S2202. 1962.

[23] Murr L.E., Rivas J.M. // Int. J. Impact Eng. 1994. Vol. 15. N 6. P. 785-795.

[24] Hayhurst C.J., Ranson H.J., Gardner D.J., Birnbaum N.K. // Int. J. Impact Engng. 1995. Vol. 17. N 1-3. P. 375-386.

[25] Harrison W., Loupias C., Outrebon P., Turland T. // Int. J. Impact Eng. 1995. Vol. 17. N 1-3. P. 369-374.

[26] Hohler V., Stilp A. // Proc. of the $12^{\text {th }}$ International Symposium on Ballistics. San Antonio, TX, 1990.

[27] Anderson C.E., Jr., Walker J.D., Bless S.J., Sharron T.R. // Int. J. Impact Eng. 1995. Vol. 17. N 1. P. 13-24. 